\title{
REQUISIITOS DE ACCESO AL MERCADO DE CANADÁ PARA GALLETAS A BASE DE MACA NEGRA
}

\section{CANADIAN MARKET ACCESS REQUIREMENTS FOR BLACK MACA COOKIES}

Reception: 2020-01-20 - Acceptance 2020-05-19

\section{Nadia Doris Benites-Jares ${ }^{1 ;}$ a}

(iD) ORCID iD 0000-0003-1390-5637

\section{Fabiola Milagros Cespedes Rosales ${ }^{1 ;}$ a}

(iD) ORCID iD 0000-0002-0140-1514

Erick Carlos Huallpatuero Ramos ${ }^{\mathbf{1} \text { a }}$

(iD) ORCID iD 0000-0001-6733-7685

1 Universidad Ricardo Palma. Facultad de Ciencias Económicas y Empresariales. Escuela Profesional de Administración de Negocios Globales.

a Estudiante de Administración de Negocios Globales.

\section{RESUMEN}

Determinar los requisitos de acceso al mercado canadiense para las galletas a base de maca negra. La investigación realizada fue no experimental. Para desarrollar el trabajo de investigacion nos basamos en el análisis de información de diferentes estudios previos. El conocimiento de los requisitos de entrada a Canadá permite la fácil inserción de las galletas elaboradas a base de maca negra. Canadá es un mercado conveniente porque sus consumidores tienen preferencias por los productos orgánicos. Las ferias internacionales son una herramienta fundamental ya que nos permite juntar al exportador con el importador.

Palabras clave: Plan; Exportación; Galletas; Maca negra; Canadá.

\section{ABSTRACT}

Develop an insertion plan for black maca cookies to the canadian market. The investigation done was not experimental. To develop this paperwork we rely on past investigations made. The development of an export plan allows the easy insertion of cookies made of back maca. Canada is a convenience market because the consumers have preference for organic products. The international fairs are fundamental tools because it allows the meet between the exporter and importer.

Keywords: Exportation; Plan; Cookies; Black maca; Canada. 


\section{INTRODUCCIÓN}

Perú es un país que tiene una amplia diversidad de productos nativos, de los cuales muy pocos son comercializados ya sea en el extranjero o incluso dentro Perú. Para impulsar el comercio internacional de Perú con otros países fue que se firmaron varios acuerdos bilaterales y multilaterales con diferentes países. Además de los acuerdos, el estado les otorga a los exportadores algunos beneficios para promover el comercio exterior como el Drawback, saldo a favor del exportador. Sin embargo, muchos exportadores no están aprovechando estos factores.

De acuerdo a Rosales (2017) considerando los escenarios en el cual el negocio evaluará la factibilidad de exportar galletas de quinua a un mercado norte americano. Se toman los siguientes puntos, se realiza un análisis del potencial exportador; análisis del mercado objetivo donde se verá el perfil del consumidor, perfil del país y un análisis de la competencia y por último se ejecutará un análisis de la gestión exportadora donde mencionaremos todos los requisitos, licencias y certificaciones que se requiera para que las galletas de maca negra ingresen al mercado Canadiense.

Según Andrade (2014), actualmente se han descubierto graves problemas con las exportaciones, por los obstáculos que las personas o empresas enfrentan, debido a la falta de capacitación en conceptos de comercialización internacional, el poco o escaso conocimiento de algunos exportadores, los problemas de financiamiento y las barreras no-arancelarias y arancelarias. Es por tal motivo, que al exportar un producto o servicio, se debe conocer, cuáles son los requerimientos para realizar dicha exportación.

Según Alcántara indica, el exdirector de la Escuela de Comercio Exterior de la Asociación de Exportadores (ADEX) Osorio, las compañías en el Perú exportan debido a un excedente de producción o porque desean ampliar y diversificar su mercado internacional. Es decir, no programan el inicio y final de sus actividades de exportación. Además, tampoco tienen una proyección a largo plazo y no tienen modelos o bases que les permitan minimizar los riesgos ante escenarios o sucesos en el mercado al cual se desea insertar sus productos. Esta controversia tiene como resultado la carencia de competitividad y productividad en la exportación (Alcántara, 2017)

Según lo mencionado por Osorio podemos decir que, si se conoce los requisitos de entrada a Canadá entonces sería factible la inserción de las galletas elaboradas a base de maca negra al mercado de Canadá.

Con este artículo, se pretende determinar los requisitos de entrada de galletas a base de maca negra al mercado de Canadá.

\section{MATERIAL Y MÉTODOS}

Se analizaron estudios de mercados en los últimos años de Perú a Canadá; primeramente se realizó la búsqueda de diferentes estudios relacionados a nuestro tema de investigación realizados en español así como en inglés, se procedió a analizar la información que se había obtenido para así poder decidir cuál era la información que nos serviría de forma específica en nuestra investigación e ir descartando el resto. Se procedió a la búsqueda de trabajos de investigación relacionadas con el tema de exportación de productos similares, se realizó una exhaustiva búsqueda de diferentes tesis peruanas así como del extranjero que fueran similares a nuestro tema de investigación; Así mismo se realizó la búsqueda de información sobre estadísticas sobre las exportaciones de productos naturales en los últimos años a Canadá y proyecciones de las exportaciones, para esto se utilizó la base de datos Siicex y Trade Map, para poder analizar el grado intercambio comercial de ambos países en cuanto a este tipo de productos. Se investigó en PromPeru sobre Superfoods y ferias internacionales más importantes para poder insertar el producto en el mercado canadiense.

\section{RESULTADOS}

\section{Análisis del potencial exportador}

\section{A. Producto a exportar}

El producto con el cual se pretende ingresar al mercado canadiense son galletas elaboradas a base de maca negra.

La galleta se llamará MAKU la cual es una galleta integral elaborada $100 \%$ con harina de maca negra. El ingrediente principal es la maca negra la cual es nativa de los andes peruanos, el cual provee de beneficios que lo caracterizan y que lo llevan a posicionarlo como un súper alimento. 
Las galletas MAKU son elaboradas con maca negra $100 \%$ orgánica, la cual desde su cultivo está libre de químicos que pueden alterar sus beneficios.

B. Identificación de las oportunidades y amenazas del mercado

Para identificar las oportunidades y amenazas del mercado que nuestro producto podría afrontar en el mercado canadiense, procedimos a realizar un FODA cruzado.

Tabla 1

Análisis FODA

\begin{tabular}{ll}
\hline FODA CRUZADO & FORTALEZA \\
\hline & (F1) Es un producto nutritivo. \\
& (F2) Fácil de elaborar. \\
& $\begin{array}{l}\text { (F3) Puede ser consumido por } \\
\text { personas celiacas. }\end{array}$ \\
OPORTUNIDADES & \multicolumn{1}{c}{ Estrategia F.O } \\
$\begin{array}{l}\text { (O1) El crecimiento de la } \\
\text { tendencia a consumir productos } \\
\text { saludables. }\end{array}$ & $\begin{array}{l}\text { (F1-O1) Aumentar la publicidad } \\
\text { para generar más ventas. }\end{array}$ \\
$\begin{array}{l}\text { (O2) Poca competencia por } \\
\text { la baja producción de galletas } \\
\text { elaboradas a base de maca. }\end{array}$ & $\begin{array}{l}\text { (F2-O2) Aumentar la producción, } \\
\text { para tomar mayor participación de } \\
\text { mercado por la poca competencia } \\
\text { existente. }\end{array}$
\end{tabular}

(O3) Leyes contra los snacks con bajo porcentaje nutritivo.

\section{AMENAZAS}

(T1) Productos substitutos con gran valor nutricional.

(T1) Personas con tiroides no pueden consumir maca.

(T3) Considerado globalmente como medicamento.
DEBILIDADES

(D1) Es un producto perecible.

(D2) El precio de la maca negra es alto.

(D3) Solo los exportadores ofrecen un sabor de galletas.

\section{Estrategia D.O}

(D1-O2) Tomar ventaja de la mínima competencia para generar rotación del producto.

(D3-01)Tomar ventaja del segmento de personas saludables, quienes tienen poder adquisitivo.

\section{Estrategia F.A}

Estrategia D.A

(F3-A1) Beneficiarnos de los substitutos que son preparados con harina de trigo, la cual contiene gluten.

Fuente: Elaboración propia.

Tabla 2

PESTEL

\begin{tabular}{|c|c|c|c|c|c|}
\hline LEGAL & POLÍTICO & ECONÓMICO & SOCIAL & TECNOLÓGICO & ECOLÓGICO \\
\hline $\begin{array}{l}\text { Parte } 13 \text { of } \\
\text { the Safe Food } \\
\text { for Canadians } \\
\text { Regulations. }\end{array}$ & & $\begin{array}{l}\text { Impedimentos } \\
\text { de comercio se } \\
\text { han reducido, y } \\
\text { la confianza y } \\
\text { demanda de los } \\
\text { consumidores sigue } \\
\text { creciendo por la } \\
\text { implementación } \\
\text { de certificados } \\
\text { obligatorios }\end{array}$ & $\begin{array}{l}\text { la demanda } \\
\text { del mercado } \\
\text { por productos } \\
\text { libres de gluten }\end{array}$ & & $\begin{array}{l}\text { Preocupación } \\
\text { por el planeta } \\
\text { y preferencia } \\
\text { en productos } \\
\text { orgánicos }\end{array}$ \\
\hline
\end{tabular}


un producto

puede ser

certificado como

un producto

orgánico si es

que ha sido

certificado por la

CFIA, la cual es el

ente acreditador

para alcanzar

los estándares

canadienses

de productos

orgánicos.

El gobierno

promueve la

calidad en los

productos, es

por eso que

tienen varias

regulaciones. la tendencia

es hacer

que más

personas se

preocupen

por su salud

Fuente: Elaboración propia.

\section{Cadena de valor}

\section{CONCLUSIONES}

$\begin{array}{ll}\text { La demanda de } & \text { las personas } \\ \text { los canadienses } & \text { se preocupan } \\ \text { por productos } & \text { por su salud }\end{array}$
aumento.
El factor ambiental es importante puesto que la tendencia del cuidado del planeta va en aumento

Para saber a detalle sobre nuestros proveedores, producción, transformación de la maca negra en galletas, y todo el proceso de comercio y exportación de las galletas de maca negra a Canadá, hemos elaborado la cadena de valor de nuestro producto. En este diagrama mostramos todas nuestras actividades y a todas la empresas y entidades involucradas en el desarrollo de las galletas de maca negra.

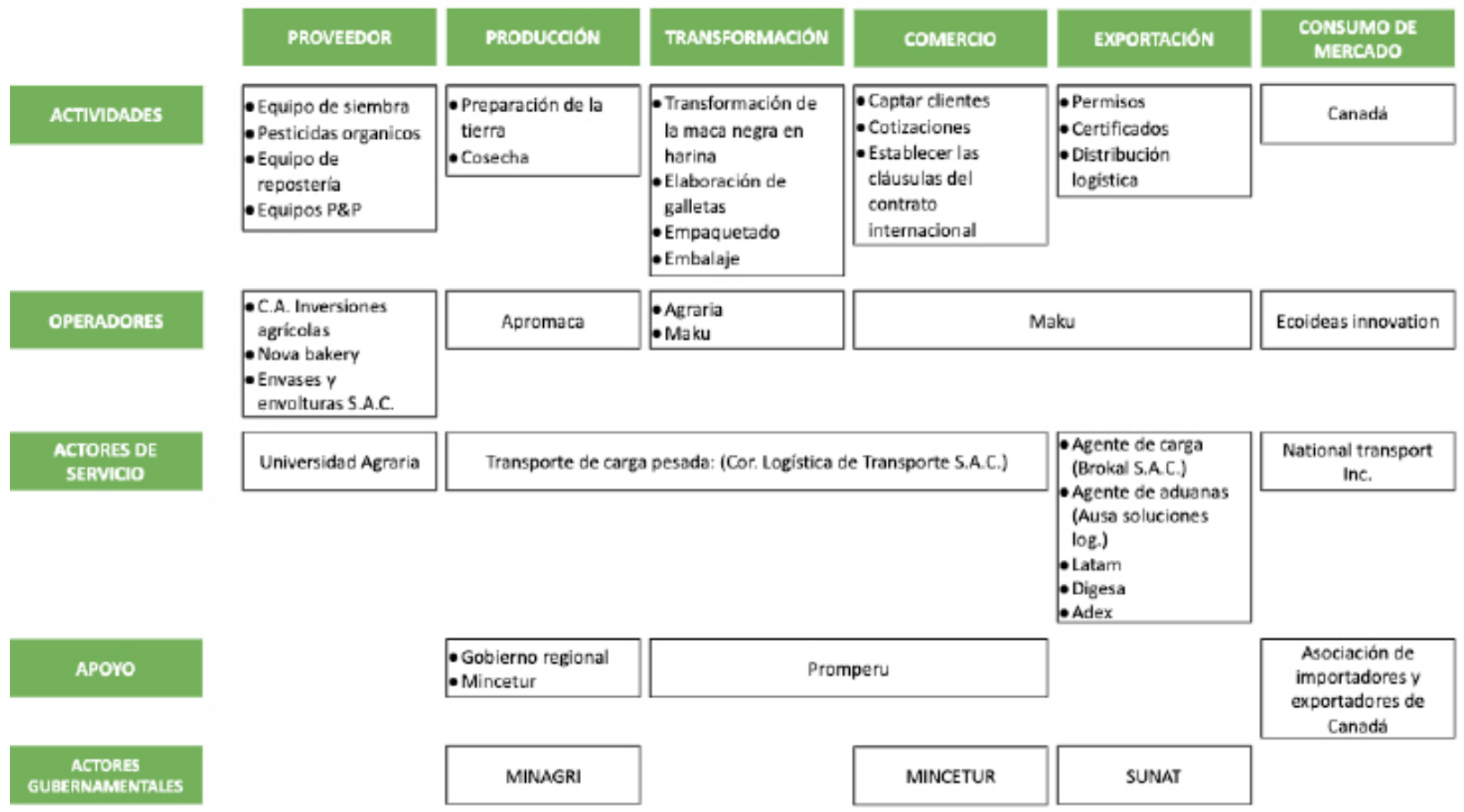

Figura 1. Cadena de valor del producto. Elaboración propia.

A. Canal de distribución

Para este plan de exportación, usaremos el canal detallista porque los puntos de venta en Canadá será en tiendas especializadas, ya que en Canadá es más factible encontrar este tipo de tiendas en todas las ciudades. Nos contactaremos con las agencias de distribución en Canadá y son estas las que se encargaran de distribuir 
nuestro producto a las diferentes cadenas de supermercados como Little Green Planet, Organic Garage, entre otros y a las tiendas especializadas. Este canal detallista es el más adecuado para llegar a nuestro público objetivo.

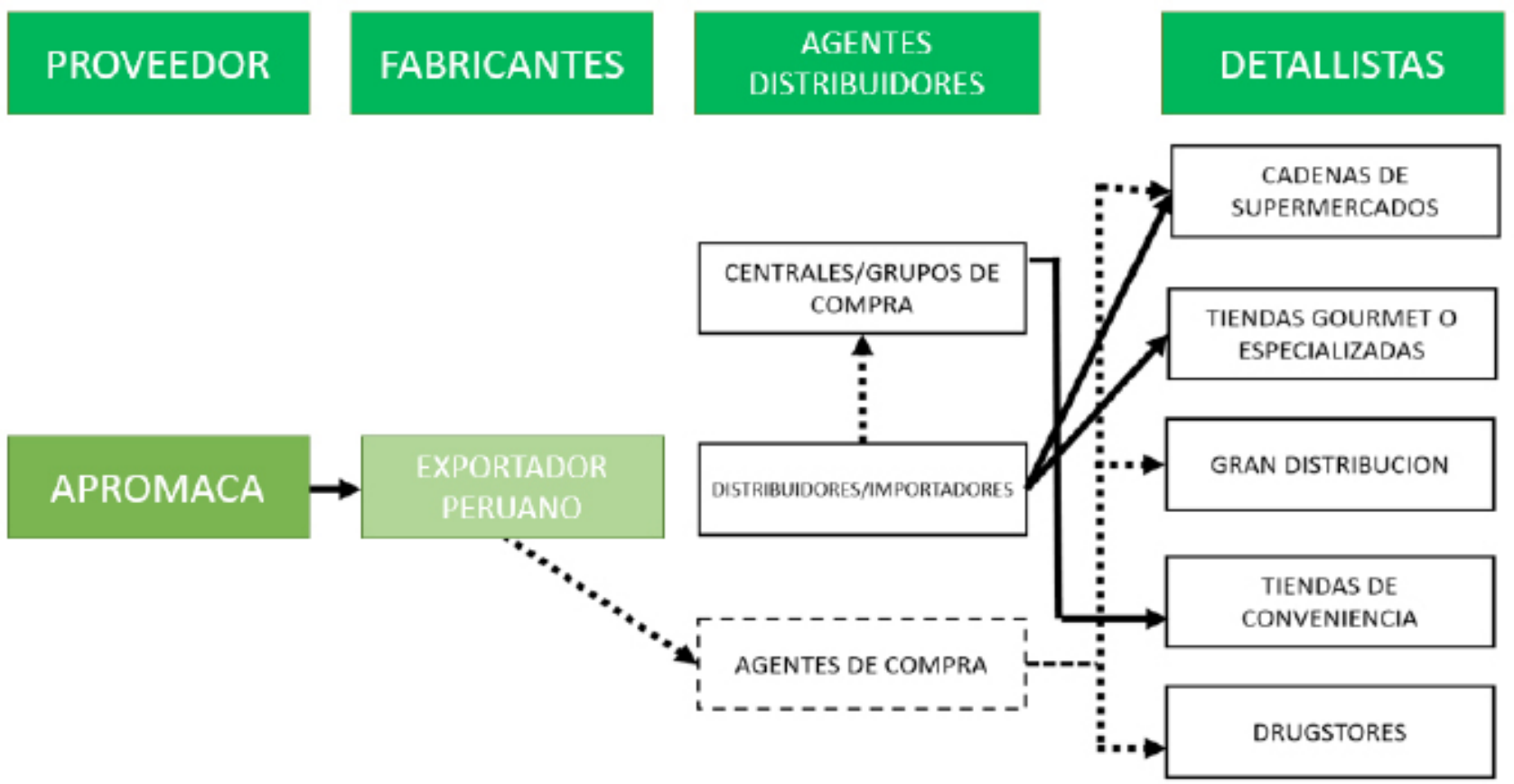

Figura 2. Canales de distribución. Elaboración propia.

\section{Canales de distribución}

1. Proveedor: Como proveedor directo se tiene a la Asociación de Productores de Maca Ecológica de la Provincia de Junín (APROMACA-E), la cual se encuentra representada legalmente por una junta directiva. Además, APROMACA-E busca el desarrollo social y económico de sus integrantes, procurando el elevar el nivel de vida de sus asociados. Las marcas de harina de maca negra que se puede encontrar en mencionada asociación son: Koken (Mix Oro), Maca Kids, Runakay.

2. Fabricante: Exportador peruano, es aquella persona natural o juridica. La cual como requisito es tener su Registro Único de Contribuyente (RUC).

3. Agentes Distribuidores: Mediante dichos agentes se entra en contacto con las cadenas de supermercados y tiendas gourmet especializadas en el país de destino (Canadá).

4. Detallistas: Son los supermercados (Gran Distribución) en Canadá, los cuales son el final de la cadena de distribución. Por ende los que tienen contacto con los consumidores finales. También entre los detallistas podemos encontrar Tiendas por Conveniencia, Drugstores.

\section{Análisis del producto en el mercado objetivo}

\section{Perfil del consumidor}

De acuerdo al Bando de desarrollo de Canadá, los consumidores canadienses están enfocados en la calidad de los productos, su origen, composición y precio, así mismo existe una tendencia de cuidado integral, que incluye al individuo, sociedad y el medio ambiente. Además tienen la disposición a pagar un precio alto si es necesario para adquirir los beneficios necesarios para su salud y bienestar. Al mismo tiempo el gobierno canadiense promueve la salud por el alto índice de personas de la tercera edad.

Es un consumidor sensible al medio ambiente y excesos de consumo; por lo tanto, buscan información de los actores que forman parte de la producción, lo social, económico, el impacto ambiental y la calidad de los productos. Toman muy en cuenta todo el proceso de la producción de los productos que están consumiendo. Tener información del producto para la trazabilidad del mismo puesto que es importante para la seguridad alimentaria. 
Asimismo el Banco de Desarrollo de Canadá acota, de que la preocupación por la salud ha ido conciencia y la conciencia por la salud está creciendo entre los habitantes de Canadá y esto se debe sobre todo por la gran cantidad de personas de la tercera edad entre su población. Esto conlleva a que los consumidores busquen nuevos productos en el mercado para satisfacer su necesidad de adquirir productos saludables que les ayuden a mejorar la salud no solo de ellos si no te toda su familia.

\section{Perfil del país}

Canadá es un mercado potencial muy importante, es por ello que queremos que nuestro producto llegue allá. Los canadienses son consumidores muy conscientes, saben lo que están consumiendo y están dispuestos a pagar un precio alto por algo que les sea de beneficio.

Para los canadienses la preocupación acerca de un estilo saludable de vida y la salud es una tendencia que se encuentra en aumento, por lo que ellos buscan productos que los ayuden a mejorar su estilo de vida; además de acuerdo con el Banco de Desarrollo de Canadá, el 70\% de los canadienses confían en las críticas hechas por otros consumidores de un producto en específico, eso indica que es muy importante para ellos buscar acerca de un producto antes de comprarlos. Asimismo, si algún producto tiene una característica de ética ambiental en su proceso de producción, los consumidores están más propensos a gastar más dinero por producto éticamente producidos y amigables con el ambiente, esta es una tendencia que está ahora mismo es su pico más alto.

\section{a. Análisis competencia local e internacional}

Algunas de las marcas representativas en Canadá son:

i) Warrior Health: Son productos a base de semillas, proteinas, chocolate y otros cereales, enfocados en un estilo de vida saludable y en forma.

ii) Crunchmaster Multi-seed crackers: Son galletas elaboradas a base de diferentes semillas como ajonjolí, chía y quinua; está certificado como libre de gluten.

iii) Lenny and Larry's: Son galletas a base proteínas, fibra veganas de diferentes presentaciones, no solo producen galletas si no ademas barras energéticas, muffins,etc.

iv) Mary's organic crackers: Son galletas elaboradas con grano entero como calabaza, chía, amapola, son libres de gluten, saborizantes, y son veganas.

Es difícil identificar nuestra competencia directa porque en el mercado de galletas peruanas la maca como ingrediente principal no es muy usado, las diferentes marcas pueden ser encontradas en las casas naturistas en toda Lima pero ninguna marca opaca a la otra.

Nuestro mayor el competidor es la compañía El Cedro, Aunque fue creada para introducir el pan árabe en Perú, pero ahora ha incrementado su oferta a diferentes productos y uno ellos es la galleta de maca. El Cedro tienen una panadería en San Isidro donde venden todos sus productos, pero sus productos más conocidos pueden ser encontrados en supermercados como Wong, Metro, Plaza Vea; tambien en tiendas de conveniencia como Listo!, Repshop, Viva!; y en ciudades como Chiclayo, Trujillo, Piura e Ica.

\section{Análisis de la gestión exportadora}

1. Requisitos

i. Análisis de peligros en puntos críticos de control (HACCP): Ayuda a los productores de alimentos procesados a verificar todos los posibles riesgos para la salud y la seguridad en la operación de sus fábricas y enfocarse en el control de los puntos críticos para la inocuidad del producto final.

ii. Buenas Prácticas de Manufactura (BPM): Ayuda a minimizar los riesgos de contaminación de un producto durante todo el proceso productivo y asegura la calidad sanitaria. Es un requisito indispensable para ofrecer productos inocuos, también es un pre requisito para cualquier sistema de calidad.

iii. Controles Previos de Importación: El importador debe tener el permiso de importación (Export and Import Controls Bureau EICB) del Ministerio de Asuntos Exteriores y Comercio Internacional de Canadá. Los permisos 
se deben tramitar con anticipación ya que solo son aceptadas 30 días antes a la fecha de llegada de la mercancía.

iv. Etiquetado: El gobierno canadiense ha cambiado como se presenta la información en los etiquetados de los productos de consumo alimenticio para que puedan ser comercializados dentro de su mercado. Las principales variaciones del etiquetado son en las especificaciones del valor nutricional del producto, así como también en los ingredientes. El etiquetado requerido para elingreso del productoal mercado canadiense se muestra más adelante.

Como se puede observar, algunos datos como los tamaños de las porciones deben ser similares a otros productos parecidos para que sea mucho más fácil para el consumidor, así mismo la cantidad de calorías debe resaltar en la información nutricional, además de la actualización de los minerales que son de preocupación para la población.

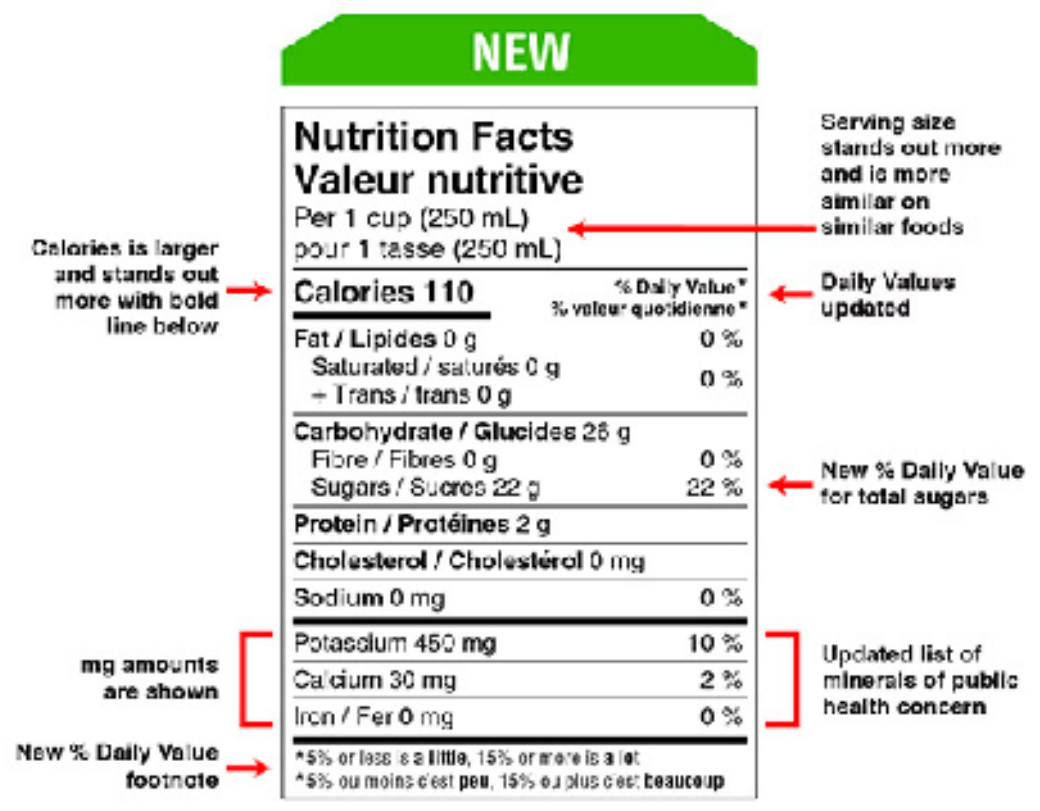

Figura 3. Datos de nutrición. Elaboración Health Canada.

En cuanto a la lista de ingredientes los más resaltante en la actualización del los etiquetados de parte del gobierno canadiense, es el listar los diferentes tipos de azúcar con los que el producto a sido elaborado.

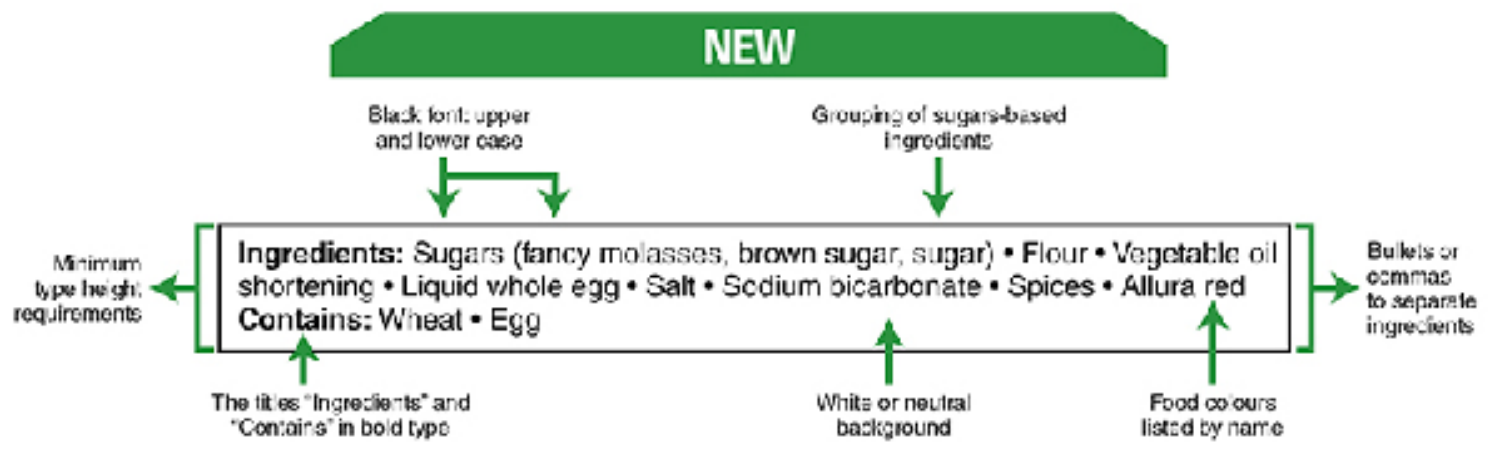

Figura 4. Lista de ingredientes. Elaboración Health Canada.

El mercado canadiense ha impuesto que los productos que vienen en unidades como las galletas o lasagna, los tamaños de porción deben ser mostrados bien en números de unidades o en porción. lo cual será emparejado como su peso en gramos. Productos similares tendrán el mismo o similar cantidad de gramos como se puede observar en la imagen de abajo. 


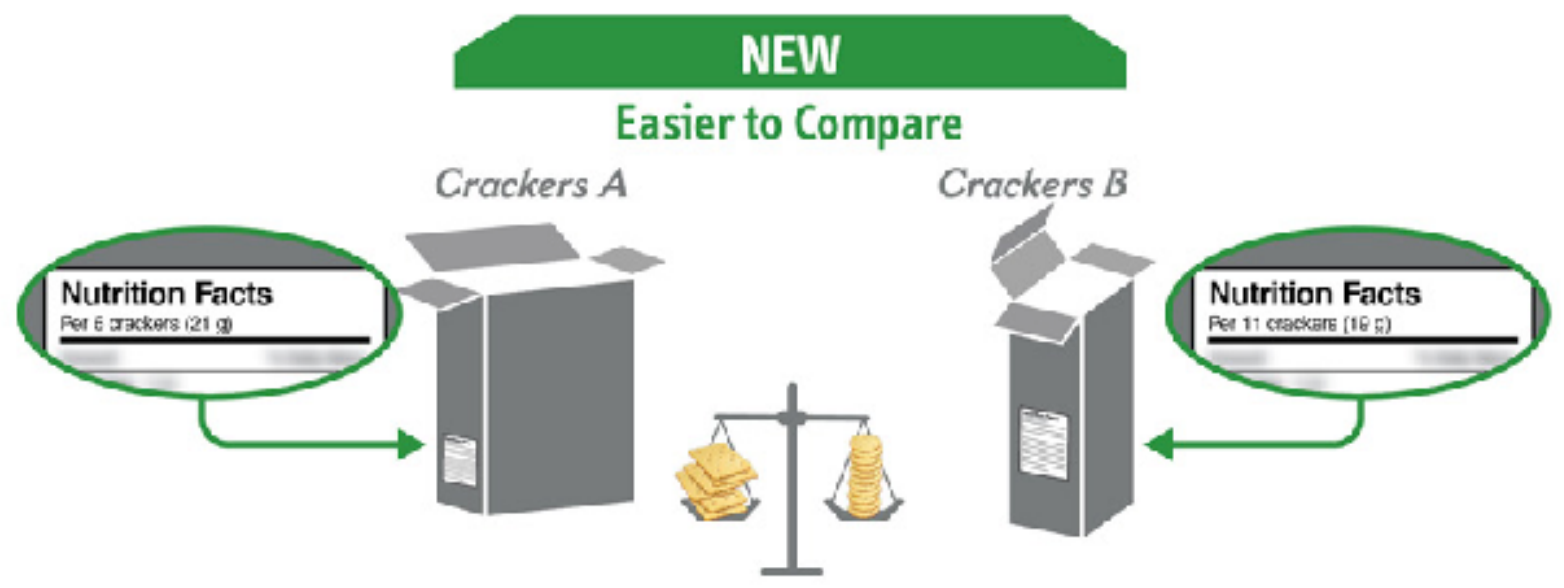

Figura 5. Alimentos que vienen en piezas o están divididas. Elaboración Health Canada.

\section{DISCUSIÓN}

El país conveniente para dirigir las exportaciones de las galletas de maca es Canadá, el cual muestra ser un país con consumidores de un alto poder adquisitivo, así mismo se caracterizan por su preferencia a los productos orgánicos. Este resultado concuerda a lo que obtenido por La Rosa \& Camaiora (2016) en el cual indica que Canadá sobre todo las ciudades del sureste poseen un mayor índice de desarrollo económico.

Se desarrolló el presente artículo con la intención de que sea una guia en cuanto a los requisitos de ingreso al mercado de Canadá para exportar galletas de maca negra o un producto con características similares al mercado canadiense, puesto que muchos exportadores no cuentan con del conocimiento o asesoría para ingresar a un mercado extranjero y no saben como ingresar a uno, este resultado se relaciona con el obtenido por Alvarado, Ramirez \& Tapia (2013) donde nos dice que el proyecto de exportación de Malanga coco a Ontario, Canadá es viable siempre y cuando se tomen en cuenta las recomendaciones y se siga correctamente el plan de negocios de exportación.

Las ferias internacionales son una importante herramienta para relacionar al productor o exportador con los intermediarios o con el consumidor final, a través de ellos se busca hacer conocida la marca o producto que se quiere se desea exportar. Este resultado concuerda con lo obtenido por Vizuete (2010), en el cual se indica que las ferias internacionales son son un instrumento fantástico ya que son eventos comerciales en los cuales se tiene la oportunidad de exhibir las cualidades de los productos y servicios.

\section{Ferias en Canadá}

De acuerdo al estudio realizado por Gil (2013) las principales ferias en el rubro alimentación son las siguientes:

Sial Canada: Es la feria más importante y representativa en el mundo. Existe una edición dirigida para el Tratado de Libre Comercio de America del Norte (TLCAN) en Toronto y Montreal.

Grocery Innovations Canada (GIC): Esta feria, está dirigida para supermercados miembros de la CFIG, se muestran productos y equipos especialmente para ellos.

Canadá Restaurant \& Foodservice Association (CRFA): Es uno de los más grandes eventos en Canadá, el sector en el cual está enfocado es hostelería. Aquí tienes la oportunidad de contactar a diferentes proveedores ya sean nacionales e internacionales.

Estas ferias nos dan la oportunidad de llegar a nuestros compradores, ya que nos permite exhibir las galletas de maca negra, mostrar los grandes beneficios que este producto tiene y tener un contacto directo con las personas interesadas en nuestro producto. 


\section{REFERENCIAS BIBLIOGRÁFICAS}

Alcantara, A. (2017). Plan de negocio para la exportación de mango Kent en el mercado de Estados Unidos. (Tesis presentada en satisfacción parcial de los requerimientos para obtener el grado de Magíster en Administración). Universidad Esan, Lima.

Alvarado, H., Ramírez, R., \& Tapia, C. (2013). Proyecto de exportación de malanga a Ontario, Canadá. Universidad Veracruzana, Veracruz.

Andrade, M. (2014). Plan de exportación de café a Hamburgo (Tesis para optar el título de licenciada en Negocio Internacionales). Universidad de América Latina, Veracruz.

APROMACA-E. (2019). La Asociación de Productores de Maca Ecológica de la Provincia de Junín. Recuperado el 26 de Noviembre de 2019, desde http://www.apromacae.com/empresa.htm

Banco de desarrollo de Canadá. (2013). Five game changing consumer trends. Recuperado el 26 de octubre de 2019, desde https://www.bdc.ca/Resources\%20Manager/study_2013/consumer_trends_BDC_report.pdf

Gil, A. (2013). El mercado de la alimentación en Canadá. Estudios de Mercado. Recuperado el 10 de noviembre de 2019, desde http://www3.icex.es/icex/cma/contentTypes/common/records/mostrarDocumento/?doc $=4722620$

La Rosa, C., \& Camaiora, C. (2016). Exportación de banano orgánico a Canadá (Tesis para optar el Título Profesional de Licenciado en Negocios Internacionales). Universidad de Lima, Lima.

Rosales, N. (2017). Exportación de galletas de quinua endulzadas con miel con diseño safari tridimensional al mercado de niños celiacos de los Ángeles-Estados Unidos (Tesis para optar el título Profesional de Licenciada en Administración de Negocios Internacionales). Universidad San Martin de Porres, Lima.

Vizuete, J. (2010). Las ferias de comercio como herramienta de mercadeo para la promoción de exportaciones (Tesis para optar ell grado de: Magíster en Comercio y Negociación Internacional). Universidad Tecnológica Equinoccial, Quito.

Correo electrónico: nadia.benites@urp.edu.pe 